\title{
El financiamiento y su influencia en la liquidez de las micro y pequeñas empresas agroexportadoras en la Región Lima Provincias 2015-2016
}

\author{
Financing and its influence on the liquidity of micro and small agro-export companies in the \\ Lima Provinces Region 2015-2016
}

M(o) CPC. Miguel Fernando Ramos Romero

\section{RESUMEN}

Objetivo: Determinar si el financiamiento de las Instituciones Financieras, influyen en la liquidez de las Micros y Pequeñas Empresas Agroexportadoras de la Región Lima Provincias. Material y Método: Se aplicó una Investigación de tipo no experimental, cuyo diseño metodológico es transaccional correlacional, con una muestra de 63 empresarios elegidos de manera aleatoria simple que se usa en variables cualitativas, y para la prueba de hipótesis se usó la distribución Chi Cuadrada, pues los datos para el análisis deben estar en forma de frecuencias. Resultados: La evaluación entre las variables Factoring (X1) y Recuperación de las cuentas por cobrar (Y1); Leasing Financiero (X2) y Programa de egresos del flujo de caja (Y2); Garantías requeridas y Fondos sujetos a restricción $(Y 3)$, obteniéndose el valor del estadístico de la tabla estadística de $C$ i Cuadrado $\mathrm{X}_{\mathrm{c}}{ }_{\mathrm{c}}=$ $31,686, X 2 c=33,343, X 2 c=27,765$, respectivamente. Conclusión: Se ha determinado que el financiamiento proporcionado por las instituciones financieras, influye positivamente en la liquidez de las Micros y Pequeñas Empresas Agroexportadoras de la Región Lima - Provincias.

Palabras Clave: Transaccional correlacional, Instituciones Financieras, Micro y pequeñas Empresas Agroexportadoras

\section{ABSTRACT}

Objective: To determine if the financing of the Financial Institutions influences the liquidity of the Micro and Small Agro-export Companies of the Lima Provinces Region. Material and Method: A non-experimental investigation was applied, whose methodological design is transactional correlational, with a sample of 63 entrepreneurs chosen in a simple random way that is used in qualitative variables, and for the hypothesis test the Chi Square distribution was used since the data for the analysis must be in the form of frequencies. Results: The evaluation between the variables Factoring (X1) and Recovery of accounts receivable (Y1); Financial Leasing (X2) and Cash Flow Disbursement Program (Y2); Required guarantees and Funds subject to restriction (Y3), obtaining the value of the statistic of the Chi Square statistical table $X 2 c=31,686, X 2 c=33,343, X 2 c=27,765$, respectively. Conclusion: It has been determined that the financing provided by financial institutions has a positive influence on the liquidity of the Micro and Small Agro-export Companies of the Lima Region - Provinces.

Key Words: Correlational Transactional, Financial Institutions, Micro and Small Agro-Export Companies

\section{NTRODUCCIÓN}

En una realidad compleja y cambiante, como la que experimenta actualmente la sociedad y en especial el sector empresarial en todos sus niveles, la tecnología, el conocimiento y las teorías cada vez más especializados han llevado a la humanidad a un desarrollo incomparable, pero al mismo tiempo se han establecido escenarios de alta competencia donde el capital, ya sea material o intelectual, juega un rol importante en el predominio de unos sobre otros. Es, en estos escenarios, donde la microempresa batalla por un lugar y un poco de recompensa.

La carencia de capitales en las microempresas es evidente, por tanto el financiamiento se hace necesario. Boscán y Sandrea. (2006), define el financiamiento: Constituye la opción con que las empresas cuentan para desarrollar estrategias de operación mediante la inversión, lo que les permite aumentar la producción, crecer, expandirse, construir o adquirir nuevos equipos o hacer alguna otra inversión que se considere benéfica para sí misma o aprovechar alguna oportunidad que se suscite en el mercado.

Mencionar a Domínguez Gómez, Efrén. (2005), "Fuentes de Financiamiento Empresarial", define al financiamiento empresarial como la obtención de recursos o medio de pago, que se destinan a la adquisición de los bienes de capital que la empresa

Recibido 24/02/2021 Aprobado 28/02/2021

Este es un artículo de acceso abierto, distribuido bajo los términos de la Licencia Creative Commons Atribución 4.0 Internacional (http://creativecommons.org/licenses/by/4.0/)

\section{(c) (i)}

M(o) CPC de la Facultad de Ciencias Económicas, Contables y Financieras de la Universidad Nacional José Faustino Sánchez Carrión. Huacho, Huaura, Lima , 1Perú.ORCID: https://orcid.org/ 0000-0002-8531-9576. Email: mramosr@unjfsc.edu.pe Pertenece al Colegio de Contadores Públicos del Callao Lic. N 1394 
necesita para el cumplimiento de sus fines.

Según su origen, las fuentes financieras suelen agruparse en financiación internas y financiación externa; también conocida como fuentes de financiamiento propias y ajenas. Sin duda los medios de financiamiento son una alternativa viable para cubrir la carencia del capital, más aún en las microempresas, pero se debe tener en cuenta que el uso de los medios de financiamiento impactará directamente en la rentabilidad, es allí donde surge el gran problema en el sector microempresa.

El impacto del financiamiento en la rentabilidad es directo, ésta puede ser positivo o negativo. Por ello Torre Padilla, Abdías Armando (2013), en su obra "Finanzas de Empresas", menciona que: La financiación no es una limosna que se da a quien la necesita, sino que es un negocio como cualquier otro. Hay que demostrar que el nuestro es un negocio, que permite a quienes prestan dinero recuperarlo y además ganar unos intereses.

Uno de los objetivos de la empresa es su crecimiento sostenido. Este crecimiento económico normalmente se da a través de proyectos de expansión que requieren de grandes inversiones, que muchas veces no cuenta la empresa para realizarlas. Sin duda pueden influir la forma, los plazos, la tasa de interés, la cantidad, la oportunidad en las cuales aplicamos el financiamiento, incluso los usos adecuados de los mismos pueden marcar la diferencia entre ganar o perder; es en esos escenarios donde se presenta un problema recurrente en las microempresas, muchos de ellos han quebrado después del financiamiento, las causas pueden ser diversas (las mismas desconozco), motivo por la que en busca de respuestas, me dispongo a investigar el financiamiento y su influencia en la liquidez de las Micro y Pequeñas empresas Agroexportadoras en la región Lima Provincias; con el objetivo de determinar el financiamiento y su influencia en la liquidez, y específicamente determinar en qué medida el financiamiento impacta en el estado de resultados y en el estado de flujo de efectivo.

El objetivo general fue determinar si el financiamiento de las instituciones financieras influye en la liquidez de las Micros y Pequeñas Empresas Agroexportadoras de la Región Lima Provincias.

Entre los objetivos específicos se determinaron: demostrar si el empleo del factoring, incide en las cuentas por cobrar de las Micros y Pequeñas Empresas Agroexportadoras de la Región Lima Provincias; demostrar que el leasing financiero, incide en Programa de egresos del flujo de caja de las Micros y Pequeñas Empresas Agroexportadoras de la Región Lima Provincias; determinar los factores para el cumplimiento de las garantías solicitadas por las instituciones financieras, incide en los Fondos Sujetos restricción de las Micros y Pequeñas Empresas Agro Exportadoras de la Región Lima Provincias.
Por último, se identificó los medios de financiamiento óptimos y adecuados para la microempresa del sector agroexportador. No hay estudio similar o igual hasta la fecha que haya determinado el grado de impacto del financiamiento en la microempresa del sector agroexportador de las Micro y Pequeñas Empresas de la Región Lima Provincias. En el trabajo de investigación no se han estudiado los perfiles de los microempresarios, ni los factores que intervienen en el buen desarrollo de la microempresa. Sólo se estudió el impacto del financiamiento y su influencia en la liquidez de las microempresas.

El trabajo de investigación es importante porque partió de un hecho concreto, de un problema que afecta a muchas empresas y en especial a las microempresas; el problema es la falta de capital y el acceso al financiamiento para muchos es la solución, sin embargo, se observa que el ciclo de vida de la microempresa es relativamente corto pese al financiamiento. Precisamente, establece el grado de impacto del financiamiento bancario en la rentabilidad. También es importante porque identifica las ventajas y desventajas del financiamiento, determino las causas por las cuales el financiamiento en algunos sectores del empresariado y de la sociedad más que ventajas representan desventajas, nos permitió tener una idea concreta y acertada de los medios de financiamiento, la oportunidad en la que se debe acceder y los plazos en las cuales la microempresa debe trabajarlo, así como identificar cual es el mejor medio de financiamiento para las microempresas.

\section{MATERIALES Y METODOS}

Esta investigación, por su finalidad es una investigación no experimental, cuyo diseño metodológico es transaccional correlacional, la población de estudio fue de 120 empresarios de la Región Lima Provincias y una muestra de 63, como técnicas de recolección de datos se empleó la encuesta y la observación. Asimismo para el procesamiento de datos estadístico del trabajo de campo realizado comprende básicamente las tablas estadísticas que corresponde a la estadística descriptiva y del contraste de las hipótesis formulada mediante la prueba no paramétrica de Chi Cuadrado, cabe indicar que el procesamiento se ha realizado en el software estadístico SPSS versión 23.

\section{RESULTADOS}

Los resultados obtenidos en relación a las variables de estudio, expresan básicamente la existencia de una relación positiva, que se demuestra en las siguientes tablas:

\section{Hipótesis $\mathrm{N}^{\circ} 1$ :}

Si las Micros y Pequeñas Empresas Agroexportadoras de la Región Lima Provincias se emplea el Factoring , entonces, habrá recuperación de las cuentas por cobrar

\section{a. Formular la hipótesis nula $\left(\mathrm{H}_{0}\right)$}


Si las Micros y Pequeñas Empresas Agroexportadoras de la Región Lima Provincias se emplea el Factoring, entonces no habrá recuperación de las cuentas por cobrar

\section{b. Formular la hipótesis alternante (Ha)}

Si las Micros y Pequeñas Empresas Agroexportadoras de la Región Lima Provincias se emplea el Factoring, entonces si habrá recuperación de las cuentas por cobrar.

\section{c. Fijar el nivel de significación ( $\alpha$ )}

Es la probabilidad de rechazar la hipótesis nula siendo verdadera, su rango de variación es $5 \% \leq \alpha \leq 10 \%$, y está asociada al valor de la tabla Ji-Cuadrado que determina el punto crítico $\left(X^{2}\right)$, específicamente el valor de la distribución es $\mathbf{X}_{\mathbf{t}(k-1),(r-1) g l .}^{2}$ y se ubica en la Tabla estadística Ji-Cuadrado, este valor divide a la distribución en dos zonas, de aceptación y de rechazo tal como indica el gráfico; si $\mathbf{X}_{\mathrm{c}}{ }_{\mathrm{c}} \mathbf{e s} \geq \mathbf{X}_{\mathrm{t}}^{2}$, entonces se rechazará la hipótes nula.

$$
X_{t}^{2}=26,296
$$

\section{d. Calcular la prueba estadística}

Se calcula con la fórmula siguiente:

Donde :

$$
x_{c}^{2}=\sum \frac{\left(0_{i}-e_{i}\right) 2}{e_{i}}
$$

$$
\begin{array}{ll}
\text { oi } & \text { Valor observado producto de las } \\
\text { encuestas }
\end{array}
$$

con datos de la muestra que se trabajan y se debe comparar con los valores paramétricos ubicados en la tabla de la Ji-Cuadrado según el planteamiento de la hipótesis alternante e indicados en el paso c.

$\mathrm{X}_{\mathrm{t}(\mathrm{kn})}^{2} \quad$ Valor del estadístico obtenido en la tabla estadística de Chi Cuadrado.

$\mathrm{K} \quad$ Filas, $\mathrm{r}=$ columnas, $\mathrm{gl}=$ grados de libertad

$X^{2}=31,686$

\section{e. Toma de decisiones}

Se debe comparar los valores de la Prueba con los valores de la tabla.

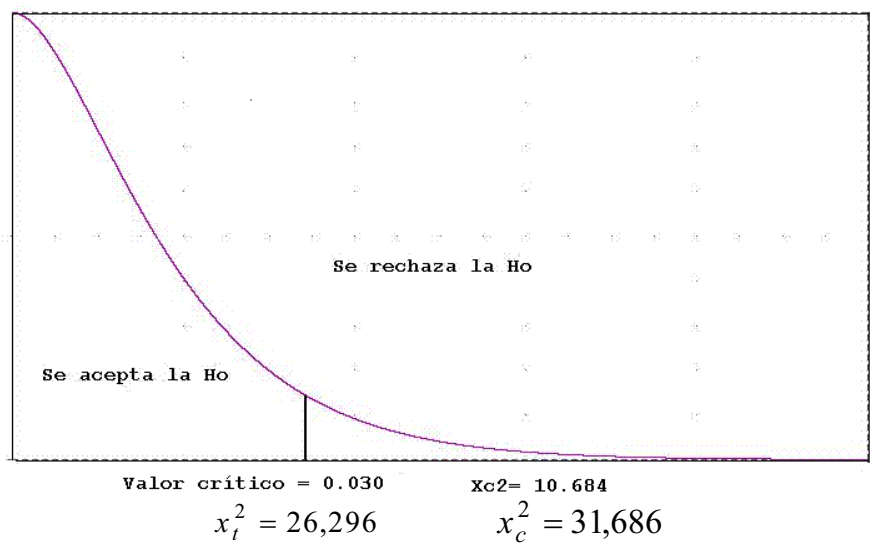

Figura. Valores de Chi cuadrado critico

\section{Tabla 1}

Si las Micros y Pequeñas Empresas Agroexportadoras de la Región Lima Provincias se emplea el Factoring, entonces, habrá recuperación de las cuentas por cobrar.

\begin{tabular}{cccccc}
\hline & & Frecuencia & Porcentaje & Porcentaje válido & Porcentaje acumulado \\
\hline Válido & Nunca & 17 & 27,0 & 27,0 & 27,0 \\
& Ocasionalmente & 26 & 41,3 & 41,3 & 68,3 \\
& Algunas veces & 9 & 14,3 & 14,3 & 82,6 \\
& Frecuentemente & 4 & 6,3 & 6,3 & 88,9 \\
& Siempre & 7 & 11,1 & 11,1 & 100,0 \\
& Total & 63 & 100 & 100 & \\
& & & &
\end{tabular}

Nota: Elaboración propia

Tabla 2

Pruebas de chi-cuadrado

\begin{tabular}{lccc}
\hline & Valor & gl & $\begin{array}{c}\text { Significación asintótica } \\
\text { (bilateral) }\end{array}$ \\
\hline Chi-cuadrado de Pearson & 31,686 & 16 & 0,011 \\
Razón de verosimilitud & 29,271 & 16 & 0,022 \\
Asociación lineal por lineal & 5,020 & 1 & 0,025 \\
N de casos válidos & 63 & & \\
\hline
\end{tabular}




\section{Conclusión}

Con un nivel de significación de $\alpha=5 \%$ se rechaza la hipótesis nula y se acepta la hipótesis alterna, concluyendo que Si las Micros y Pequeñas Empresas Agroexportadoras de la Región Lima Provincias se emplea el Factoring, entonces si habrá recuperación de las cuentas por cobrar, dicha hipótesis ha sido probada usando la prueba estadística Chi Cuadrado y en Software estadístico SPSS, versión 23, para tal efecto se adjuntan las evidencias del caso consistente en la tabla de Cruzada $N^{\circ} 01$ y el resultado de la prueba estadística.

\section{Hipótesis 2:}

Si se usa el Leasing Financiero, entonces incide positivamente en Programa de egresos del flujo de caja de las Micros y Pequeñas Empresas Agroexportadoras de la Región Lima Provincias.

\section{a. Formular la hipótesis nula $\left(\mathrm{H}_{0}\right)$}

Si se usa el Leasing Financiero, entonces no incide positivamente en Programa de egresos del flujo de caja de las Micros y Pequeñas Empresas Agroexportadoras de la Región Lima Provincias.

\section{b. Formular la hipótesis alternante $\left(\mathrm{H}_{\mathrm{a}}\right)$}

Si se usa el Leasing Financiero, entonces si incirpositivamente en Programa de egresos del flujo de ca de las Micros y Pequeñas Empresas Agroexportador: de la Región Lima Provincias.

\section{c. Fijar el nivel de significación ( $\alpha$ ).}

Es la probabilidad de rechazar la hipótesis nula sienı verdadera, su rango de variación es $5 \% \leq \alpha \leq 10 \%$, y es asociada al valor de la tabla Ji-Cuadrado que determiı el punto crítico $\left(\mathrm{X}_{\mathrm{t}}^{2}\right.$, específicamente el valor de distribución es $\mathrm{X}_{\mathrm{t}(\mathrm{k}-1)(\mathrm{r}-1) \mathrm{gl} \text {. }}^{2}$ y se ubica en la Tabla estadísti Ji-Cuadrado, este valor divide a la distribución en di zonas, de aceptación y de rechazo tal como indica e gráfico; si $X^{2}{ }_{c}$ es $\geq X^{2}$, entonces se rechazará la hipótesis nula.

$$
X_{t}^{2}=26,296
$$

\section{d. Calcular la prueba estadística}

Se calcula con la fórmula siguiente:

Donde :

$$
X_{c}^{2}=\sum \frac{\left(o_{i}-e_{i}\right)^{2}}{e_{i}}
$$

oi Valor observado producto de las encuestas

ei Valor esperado obtenido a partir del valor esperado.

$\mathrm{X}_{\mathrm{c}}^{2} \quad$ Valor del estadístico calculado con datos de la muestra obtenidos en las encuestas.

$\mathrm{X}_{\mathrm{t}(\mathrm{kn})}^{2} \quad$ Valor del estadístico obtenido en la tabla estadística de Chi Cuadrado, siendo K Filas, $r$ = columnas, $\mathrm{gl}=$ grados $\mathrm{de}$ libertad

$$
\mathbf{X}_{\mathrm{c}}^{2}=33,343
$$

\section{a. Toma de decisiones}

Se debe comparar los valores de la Prueba con los valores de la tabla.

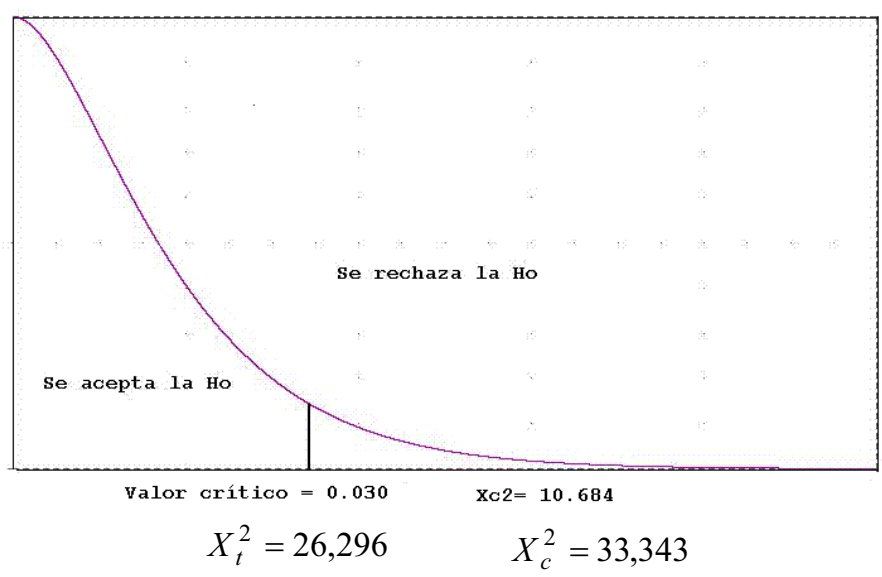

Figura. Valores de Chi cuadrado critico

\section{Tabla 3}

Si se usa el Leasing Financiero, entonces incide positivamente en Programa de egresos del fujo de caja de las Micros y Pequeñas Empresas Agroexportadoras de la Región Lima Provincias

\begin{tabular}{cccccc}
\hline & Frecuencia & Porcentaje & Porcentaje válido & Porcentaje acumulado \\
\hline Válido & Nunca & 16 & 25,4 & 25,4 & 25,4 \\
& Ocasionalmente & 24 & 38,1 & 38,1 & 63,5 \\
& Algunas veces & 9 & 14,3 & 14,3 & 77,8 \\
& Frecuentemente & 5 & 7,9 & 7,9 & 85,7 \\
& Siempre & 9 & 14,3 & 14,3 & 100 \\
& Total & 63 & 100 & 100 & \\
\hline
\end{tabular}

Nota: Elaboración propia 
Tabla 4

Pruebas de chi-cuadrado

\begin{tabular}{lccc}
\hline & Valor & gl & $\begin{array}{c}\text { Significación asintótica } \\
\text { (bilateral) }\end{array}$ \\
\hline Chi-cuadrado de Pearson & 33,343 & 16 & 0,007 \\
Razón de verosimilitud & 30,367 & 16 & 0,016 \\
Asociación lineal por lineal & 4,552 & 1 & 0,033 \\
N de casos válidos & 63 & & \\
\hline
\end{tabular}

\section{Conclusión}

Con un nivel de significación de $\alpha=5 \%$ se rechaza la hipótesis nula y se acepta la hipótesis alterna, concluyendo que Si se usa el Leasing Financiero, entonces si incide $\mathrm{Si}$ se usa el Leasing Financiero, entonces no incide positivamente en Programa de egresos del flujo de caja de las Micros y Pequeñas Empresas Agroexportadoras de la Región Lima Provincias en Programa de egresos del flujo de caja de las Micros y Pequeñas Empresas Agroexportadoras de la Región Lima Provincias, dicha hipótesis ha sido probada usando la prueba estadística Chi Cuadrado y en Software estadístico SPSS, versión 23, para tal efecto se adjuntan las evidencias del caso consistente en la tabla de Cruzada $N^{\circ} 03$ y el resultado de la prueba estadística.

\section{Hipótesis 3:}

Si se obtienen garantías requeridas por las Instituciones Financieras, entonces incide favorablemente en los Fondos Sujetos restricción de las Micros y Pequeñas Empresas Agro Exportadoras de la Región Lima Provincias.

\section{a. Formular la hipótesis nula $\left(\mathrm{H}_{0}\right)$}

Si se obtienen garantías requeridas por las Instituciones Financieras, entonces no incide favorablemente en los Fondos Sujetos restricción de las Micros y Pequeñas Empresas Agro Exportadoras de la Región Lima Provincias.

\section{b. Formular la hipótesis alternante $\left(\mathrm{H}_{\mathrm{a}}\right)$}

Si se obtienen garantías requeridas por la Instituciones Financieras, entonces si incid favorablemente en los Fondos Sujetos restricción d las Micros y Pequeñas Empresas Agro Exportadora de la Región Lima Provincias.

\section{c. Fijar el nivel de significación ( $\alpha$ ).}

Es la probabilidad de rechazar la hipótesis nul siendo verdadera, su rango de variación es $5 \% \leq \alpha$ $10 \%$, y está asociada al valor de la tabla Ji-Cuadrad que determina el punto crítico $\left(\mathrm{X}_{\mathrm{t}}^{2}\right.$, específicamente el valor de la distribución es $\mathbf{X}_{\mathbf{t}(k-1),(r-1) g !}\left(\mathbf{X}^{2}\right.$ se ubica en la Tabla estadística Ji-Cuadrado, este valor divide a la distribución en dos zonas, de aceptación y de rechazo tal como indica el gráfico; si $\mathbf{X}_{\mathrm{c}}^{2}$ es $\geq \mathbf{X}_{\mathrm{t}}^{2}$, entonces se rechazará la hipótesis nula.

$$
X_{t}^{2}=26,296
$$

\section{d. Calcular la prueba estadística}

Se calcula con la fórmula siguiente:

Donde :

$$
X_{c}^{2}=\sum \frac{\left(o_{i}-e_{i}\right)^{2}}{e_{i}}
$$

oi Valor observado producto de las encuestas

ei Valor esperado obtenido a partir del valor esperado.

$\mathrm{X}^{2} \quad$ Valor del estadístico calculado con datos de la muestra obtenidos en las encuestas.

$\mathrm{X}_{\mathrm{t}(\mathrm{kn})}^{2} \quad$ Valor del estadístico obtenido en la tabla estadística de Chi Cuadrado, siendo $K$ Filas, $r=$ columnas, $g l=$ grados $d e$ libertad

$$
X_{C}^{2}=27,765
$$

a.

\section{Toma de decisiones}

Se debe comparar los valores de la Prueba con los valores de la tabla.

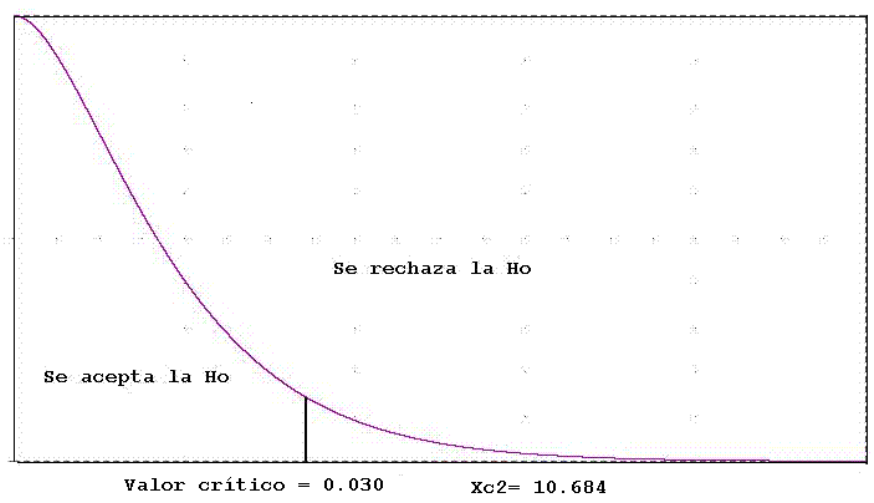

Figura : Valores de Chi cuadrado critico 
Tabla 5

\begin{tabular}{cccccc}
\hline & Frecuencia & Porcentaje & $\begin{array}{l}\text { Porcentaje } \\
\text { válido }\end{array}$ & $\begin{array}{c}\text { Porcentaje } \\
\text { acumulado }\end{array}$ \\
\hline Válido & Nunca & 22 & 34,9 & 34,9 & 34,9 \\
& Ocasionalmente & 16 & 25,4 & 25,4 & 60,3 \\
& Algunas veces & 8 & 12,7 & 12,7 & 73 \\
& Frecuentemente & 11 & 17,5 & 17,5 & 90,5 \\
Siempre & 6 & 9,5 & 9,5 & 100 \\
& Total & 63 & & 100 & \\
\hline
\end{tabular}

Nota: Elaboración propia

Tabla 6

Pruebas de chi-cuadrado

\begin{tabular}{lccc}
\hline & Valor & gl & Significación asintótica (bilateral) \\
\hline Chi-cuadrado de Pearson & 27,765 & 16 & 0,034 \\
Razón de verosimilitud & 29,354 & 16 & 0,022 \\
Asociación lineal por lineal & 1,353 & 1 & 0,0245 \\
$\mathrm{~N}$ de casos válidos & 63 & & \\
\hline
\end{tabular}

\section{Conclusión}

Con un nivel de significación de $\alpha=5 \%$ se rechaza la hipótesis nula y se acepta la hipótesis alterna, concluyendo que si se obtienen garantías requeridas por las Instituciones Financieras, entonces si incide favorablemente en los Fondos Sujetos restricción de las Micros y Pequeñas Empresas Agro Exportadoras de la Región Lima Provincias, dicha hipótesis ha sido probada usando la prueba estadística Chi Cuadrado y en Software estadístico SPSS, versión 23, para tal efecto se adjuntan las evidencias del caso consistente en la tabla de Cruzada $\mathrm{N}^{\circ} 05$ y el resultado de la prueba estadística.

\section{DISCUSIÓN}

Para realizar la discusión del presente trabajo de investigación se consideró básicamente los siguientes aspectos: el resultado de las encuestas, las entrevistas, el contraste de las hipótesis y los autores que tratan el tema objeto de la investigación conformado por las variables financiamiento y liquidez.

Considerando las encuestas, tenemos que se analizó la variable financiamiento realizando la pregunta si la empresa donde labora tiene financiamiento de las instituciones financieras, a lo que los encuestados manifestaron: el $30,2 \%$ de las personas encuestadas señaló que nunca, mientras que el $38,1 \%$ indicó que ocasionalmente, el $7,9 \%$ de las personas encuestadas señaló que algunas veces, por lo tanto el 9,52\% de las personas encuestadas respondió frecuentemente, y finalmente el $14,29 \%$ de las personas encuestadas respondió la alternativa siempre. Esto indica que las Mypes muy poco acceden a las instituciones para obtener su financiamiento, aspecto que deben tener en cuenta para incorporar en su plan de trabajo. Ello se corrobora con el planteamiento de la hipótesis principal que plantea que "El financiamiento influye positivamente en la liquidez de las Micros y Pequeñas Empresas Agroexportadoras de la Región Lima - Provincias", permitiendo a las empresas desarrollar proyectos sostenibles con producción uniforme y brindar servicios de calidad a la comunidad internacional.

La variable liquidez también formó parte de la encuesta, cuya pregunta era saber si las empresas agroexportadores disponen de la liquidez necesaria para realizar sus operaciones de negocios en la Región de Lima Metropolitana. Al respecto, el $22,2 \%$ de las personas encuestadas señaló que nunca, mientras que el $42,9 \%$ indicó que ocasionalmente, también el 12,7\% de las personas que se encuestaron respondieron que algunas veces, el $12,7 \%$ de las personas respondieron frecuentemente, finalmente el 9,5\% respondieron siempre a esta inquietud, este aspecto también se refuerza con el resultado de la hipótesis principal.

Referente a los diferentes autores se tiene, entre otros, a Gomero (2003) en su libro "Las Instituciones Financieras Intermediarias son estrategias de financiamiento a mediano plazo en las micro y pequeñas empresas Agro exportadoras del país". Indica, además, que: Las PYMES han ganado y acumulado fortalezas en el mercado a base de iniciativa e ingenio empresarial, por eso se le conoce como micro emprendedores, pero este esfuerzo ha estado lejos de ser complementado, por organizaciones, como los intermediarios financieros especialmente los bancos, quienes lo han considerado como marginal en su cartera de colocaciones, basando este hecho en el argumento del riesgo y el costo.

Así mismo Saldaña (2009), en su trabajo "EI comportamiento de la economía y la gestión de riesgos de los intermediarios financieros bancarios: caso Perú", en el que menciona que los bancos juegan un rol importante en el comportamiento económico de un país. La canalización de fondos que se realiza a través de estas instituciones permite que empresas puedan alcanzar sus objetivos económicos.

Un caso relevante es el de las empresas, que consideran 
a estas instituciones financieras como sus socios estratégicos, por el hecho de ser los proveedores de fondos líquidos que utilizan para financiar su capital de trabajo y de inversión.

De acuerdo a los resultados obtenidos en el trabajo de campo y la contratación de las hipótesis, se ha determinado que el financiamiento proporcionado por las instituciones financieras, influye positivamente en la liquidez de las Micros y Pequeñas Empresas Agroexportadoras de la Región Lima - Provincias, lo que les permite realizar proyectos sostenibles en este segmento empresarial. Así mismo se infiere que las mencionadas empresas pasan a ser formales trayendo beneficios tanto al fisco como a trabajadores.

\section{AGRADECIMIENTOS}

Mi agradecimiento a la Universidad de San Martín de Porres, en especial a las autoridades, docentes de la Escuela de Postgrado de Ciencias Contables Económicas y Financieras quienes con sus consejos me permitieron alcanzar el presente logro; así mismo a sus colaboradores administrativos que prestan un servicio de primer nivel.

Mi agradecimiento especial a mis asesores temáticos y metodológicos, quienes fueron fundamentales en la elaboración de la presente trabajo por su rol de apoyo a través de sus amplias experiencias y conocimientos.

\section{REFERENCIAS BIBLIOGRAFICAS}

\section{Fuentes Bibliográficas}

Boscán y Sandrea. (2006). Estrategias de financiamiento para el desarrollo del sector confección. Zuliano. Revista de Estudios Interdisciplinarios en Ciencias Sociales. Volumen 11. $N^{\circ} 3$.

Domínguez, E. (2005). Trabajo de Diploma en opción al título de licenciado en economía. Propuesta de una alternativa de financiamiento para el Hotel Playa de Oro Varadero. México: Universidad de Matanzas.

Gomero, N. (2003). Participación de los intermediarios financieros bancarios y no bancarios en el desarrollo de la Pymes textiles en Lima Metropolitana. Lima. Tesis Universidad de Mayor de San Marcos.

Saldaña, J (2009). El Comportamiento de la economía y la gestión de riesgos de los intermediarios Financieros Bancarios: caso Perú: 2001-2006. Tesis de Maestro. Universidad Nacional Mayor de San Marcos. Lima.

Torre, A. (2013). Casos prácticos de finanzas de empresa. Universidad Nacional del Callao. 